\title{
Imaging of Mesenchymal Stem Cell Transplant by Bioluminescence and PET
}

Zachary Love ${ }^{1}$, Fangjing Wang 2 , James Dennis*3,4, Amad Awadallah ${ }^{3}$, Nicolas Salem ${ }^{2}$, Yuan Lin ${ }^{5}$, Andrew Weisenberger ${ }^{6}$, Stan Majewski ${ }^{6}$, Stanton Gerson ${ }^{5,4}$, and Zhenghong Lee*1,2,4

${ }^{I}$ Department of Nuclear Medicine/Radiology, University Hospitals Case Medical Center, Case Western Reserve University, Cleveland, Ohio; ${ }^{2}$ Department of Biomedical Engineering, Case Western Reserve University, Cleveland, Ohio; ${ }^{3}$ Department of Orthopaedics, Case Western Reserve University, Cleveland, Ohio; ${ }^{4}$ Center for Stem Cell and Regenerative Medicine, Case Western Reserve University, Cleveland, Ohio; ${ }^{5}$ Department of Hematology/Oncology, Case Western Reserve University, Cleveland, Ohio; and ${ }^{6}$ Thomas Jefferson National Accelerator Facility, Newport News, Virginia

Dynamic measurements of infused stem cells generally require animal euthanasia for single-time-point determinations of engraftment. In this study, we used a triple-fusion reporter system for multimodal imaging to monitor human mesenchymal stem cell (hMSC) transplants. Methods: hMSCs were transduced with a triple-fusion reporter, fluc-mrfp-ttk (encoding firefly luciferase, monomeric red fluorescent protein, and truncated herpes simplex virus type 1 sr39 thymidine kinase) by use of a lentiviral vector. Transduced cells were assayed in vitro for the expression of each functional component of the triple-fusion reporter. Transduced and control hMSCs were compared for their potential to differentiate into bone, cartilage, and fat. hMSCs expressing the reporter were then loaded into porous, fibronectin-coated ceramic cubes and subcutaneously implanted into NOD-SCID mice along with cubes that were loaded with wild-type hMSCs and empty cubes. Mice were imaged repeatedly over 3 mo by bioluminescence imaging (BLI), and selected animals underwent CT and PET imaging. Results: Osteogenic, adipogenic, and chondrogenic potential assays revealed retained differentiation potentials between transduced and wild-type hMSCs. Signals from the cubes loaded with reporter-transduced hMSCs were visible by BLI over 3 mo. There was no signal from the empty or wild-type hMSC-loaded control cubes. PET data provided confirmation of the quantitative estimation of the number of cells at one spot (cube). Cubes were removed from some animals, and histologic evaluations showed bone formation in cubes loaded with either reporter-transduced or wild-type hMSCs, whereas empty controls were negative for bone formation. Conclusion: The triple-fusion reporter approach resulted in a reliable method of labeling stem cells for investigation in small-animal models by use of both BLI and small-animal PET imaging. It has the potential for translation into future human studies with clinical PET.

Received May 1, 2007; revision accepted Sep. 11, 2007.

For correspondence or reprints contact either of the following:

Zhenghong Lee, Department of Nuclear Medicine/Radiology, University

Hospitals Case Medical Center, 11100 Euclid Ave., Cleveland, OH 44106.

E-mail: zhenghong.lee@case.edu

James Dennis, Department of Orthopaedics, Case Western Reserve

University, 11100 Euclid Ave., Cleveland, $\mathrm{OH} 44106$.

E-mail: james.dennis@case.edu

${ }^{*}$ Contributed equally to this work.

COPYRIGHT @ 2007 by the Society of Nuclear Medicine, Inc.
Key Words: bioluminescence imaging; PET; mesenchymal stem cells; triple-fusion reporter genes

J Nucl Med 2007; 48:2011-2020

DOI: 10.2967/jnumed.107.043166

$\mathbf{T}$ here is increasing evidence that adult human tissues harbor stem and progenitor cells that can be used for therapeutic purposes. Human mesenchymal stem cells or multipotent marrow stromal cells (hMSCs) are self-renewing, pluripotent adult stem cells that are found in adult donor bone marrow and that can differentiate into bone, cartilage, and adipose tissues (1-3). Autologous and allogeneic transplantation of hMSCs has been reported to have many tantalizing effects and potentials in the treatment of human diseases. For example, in bone marrow transplant recipients, intravenously administered hMSCs have been reported to promote engraftment of hematopoietic lineages in cancer patients receiving high-dose chemotherapy (4) and to ameliorate graft-versus-host disease $(5,6)$. Furthermore, intravenously administered hMSCs have been shown to home to neoplasms in vivo and thus have the potential to serve as vehicles for the delivery of anticancer therapies (7). Finally, hMSCs injected in the setting of myocardial infarction and stroke improve postischemic regeneration and function $(8,9)$.

The promise of MSC therapies mandates research leading to a better understanding of the long-term fate and trafficking of transplanted MSCs in vivo. Previously, the majority of such research relied on ex vivo labeling of MSCs by various methods, performing transplants, and euthanizing animals at serial posttransplant time points to search for histologic evidence of the fate of MSCs. This methodology has the obvious drawback of making the understanding of the longitudinal fate of the transplant within a given recipient impossible. For this reason, we investigated the feasibility of a noninvasive, longitudinal approach to the monitoring of allogeneic hMSC transplants in vivo.

Recently, several noninvasive, imaging-based monitoring methods were used to track MSC transplants. These methods 
include ex vivo, direct labeling of cells for transplantation with iron oxide particles or perfluoropolyether for MRI $(10,11)$, with ${ }^{18} \mathrm{~F}$-hexafluorobenzene or ${ }^{18} \mathrm{~F}$-FDG for PET $(12,13)$, or with ${ }^{111} \mathrm{In}$-oxine or ${ }^{111} \mathrm{In}$-tropolone for SPECT (14-16). Direct labeling of MSCs with radionuclides only allows imaging of the initial deposition of infused MSCs; the imaging signal decreases or becomes more diffusive with radiodecay, cell division, and cell death. Indirect labeling relies on the expression of imaging reporter genes transduced into cells before transplantation; the genes are then visualized on infusion of appropriate probes. Examples of this approach include labeling with the firefly luciferase gene $(f l u c)$ for bioluminescence imaging (BLI), the herpes simplex virus type 1 (HSV1) thymidine kinase gene $(t k)$ for PET imaging (17-19), or the transferrin receptor gene (TfR) for nuclear imaging or MRI (20). The reporter gene fluc results in a high sensitivity of BLI in small-animal models but will be difficult to translate into clinical use because of the constraints of tissue penetration of visible-light photons. However, the reporter gene $t k$ allows quantitative PET and has the potential for translation into a clinical setting.

In this study, we transduced hMSCs with a reporter system that allowed for both qualitative imaging and quantitative imaging of transplants in vivo in small-animal models longitudinally. Specifically, we used the triple-fusion reporter fluc-mrfp-ttk (LRT; a gift from Sam S. Gambhir, Stanford University), encoding a fusion protein containing functional components from firefly luciferase ( $f u \mathrm{c})$, monomeric red fluorescent protein (mrfp), and truncated HSV1 sr39 thymidine kinase (ttk); the products of these genes can be visualized with BLI, fluorescence imaging, and PET imaging, respectively (21). The individual components or domains were linked by 14-amino-acid (LENSHASAGYQAST) or 8 -amino-acid (TAGPGSAT) segments. The $t t k$ domain contains a deletion in the first 135 base pairs that eliminates the nuclear localization signal. As a result, higher cytosolic than nuclear concentrations result in less cytotoxicity and an improved image signal. The enzymatic activities of the domains in this fusion reporter were previously shown to be $54 \%$ for $f l u c$, "medium" for $m r f p$, and $100 \%$ for $t t k$ when compared with those of their full-length individual counterparts. We chose this fusion reporter with undiminished thymidine kinase activity in the hope that maximizing the potential for PET imaging would be most useful in future clinical studies. Although this and similar reporters have been used elsewhere in the context of stem cell transplants (21-24), our study is unique in its use of a secondgeneration lentiviral vector for reporter gene transduction, in the use of native hMSCs, and in the thorough examination of the effects of the reporter on the stem cell phenotype. In particular, the differences between this study and another MSC tracking study (19) were as follows: this study was a general-purpose investigation of an imaging-based MSC tracking technique, whereas the other study focused on tumor metastasis tracking; primary hMSCs were used in this study instead of immortalized MSCs; a triple-fusion reporter ( $f u c-m r f p-t t k)$ was used in this study instead of a dual-fusion reporter (egfp-tk), and the addition of $f l u c$ allowed convenient and sensitive BLI; and a modified myeloproliferative sarcoma virus promoter (mnd promoter) was used to drive triple-fusion reporter expression, as it was shown to be more resistant to epigenetic silencing (25) than the elongation factor $1 \alpha$ promoter.

\section{MATERIALS AND METHODS}

\section{Lentiviral Vector Construction}

The triple-fusion reporter $f u c-m r f p$ - $t$ t , contained in the construct pCDNA3.1-CMV-triplefusion (from Sam S. Gambhir, Stanford University), was inserted into the second-generation, self-inactivating lentiviral vector $\mathrm{pHR}^{\prime}$-mnd-LINK14, which drives reporter transcription with a modified myeloproliferative sarcoma virus promoter (mnd promoter) to produce the plasmid pHR'-mnd-fluc-erfp-ttk. In brief, a NotI site was created in the second-generation, self-inactivating lentiviral vector $\mathrm{pHR}^{\prime}$-mndLINK14 by insertion of the oligonucleotide 5'-GCGGCCGCGTAC-3' into the KpnI site to yield the vector $\mathrm{pHR}^{\prime}$-mnd-Not. A NotI site was also introduced into the construct pCDNA3.1-CMVtriplefusion at the NheI site upstream of the reporter by use of the oligonucleotide 5'-CTAGGCGGCCGC-3'. The NotI fragment containing the reporter was then ligated into the NotI site in the vector to yield the vector $\mathrm{pHR}^{\prime}$-mnd-LRT.

\section{Transient Transfection}

Infectious virus was produced by triple transient cotransfection of 293T/17 cells (American Type Culture Collection) with pHR'mnd-LRT, pCMV $\Delta$ R8.91 (packaging vector), and pMD.G (vesicular stomatitis virus protein $\mathrm{G}$ pseudotyping vector) as described by Zielske et al. (26). Additionally, infectious virus lacking the reporter (empty vector) was created by identical transfection but with $\mathrm{pHR}^{\prime}$-mnd-Not substituted for $\mathrm{pHR}^{\prime}$-mnd-LRT. In brief, confluent $293 \mathrm{~T} / 17$ cells from a 100-mm plate were used to seed a $175-\mathrm{cm}^{2}$ flask containing $25 \mathrm{~mL}$ of $293 \mathrm{~T}$ growth medium (Dulbecco's modified Eagle medium [DMEM; Invitrogen], glucose $[4.5 \mathrm{~g} / \mathrm{L}], 10 \%$ fetal bovine serum [FBS; Gibco], $2 \mathrm{mM}$ GlutaMAX [Invitrogen], penicillin [100 U/mL; Invitrogen], and streptomycin [100 $\mu \mathrm{g} / \mathrm{mL}$; Invitrogen]) $24 \mathrm{~h}$ before transfection. All cell cultures were incubated at $37^{\circ} \mathrm{C}$ under $5 \%$ carbon dioxide. Transfection was performed with serum-free OptiMEM I (Invitrogen) by use of Lipofectamine 2000 (Invitrogen) according to the manufacturer's instructions. The mass ratio of $\mathrm{pHR}^{\prime}$-mnd-LRT to pCMV $\Delta$ R8.91 to pMD.G was 3:3:1, respectively. After $24 \mathrm{~h}$ of transfection, OptiMEM I was replaced with $25 \mathrm{~mL}$ of hMSC growth medium (DMEM, glucose $[1 \mathrm{~g} / \mathrm{L}], 10 \%$ FBS selected for use with hMSCs as previously described by Lennon et al. (27) FBS, $2 \mathrm{mM}$ GlutaMAX, penicillin $[100 \mathrm{U} / \mathrm{mL}$ ], and streptomycin [100 $\mu \mathrm{g} / \mathrm{mL}]$ ), and incubation was continued for an additional $48 \mathrm{~h}$. Supernatant containing virus was collected and sterilized with $0.22-\mu \mathrm{m}$ Steriflip (Millipore) filters before storage at $-80^{\circ} \mathrm{C}$.

Titers were determined by transduction of K562 cells (American Type Culture Collection) followed by permeabilization, immunolabeling for firefly luciferase, and flow cytometry. In brief, $5 \times 10^{5} \mathrm{~K} 562$ cells were used to seed each of 5 wells of a 6-well plate containing $2 \mathrm{~mL}$ of K562 growth medium [Iscove's modified Eagle medium [Cellgro], 10\% hMSC-tested FBS [Gibco], $2 \mathrm{mM}$ GlutaMAX, penicillin [100 U/mL], and streptomycin $[100 \mu \mathrm{g} / \mathrm{mL}])$. Virus was thawed, and $0,50,100,200$, or 
$400 \mu \mathrm{L}$ were brought to a volume of $500 \mu \mathrm{L}$ in $\mathrm{K} 562$ growth medium before being added to the cells. Polybrene (Sigma Chemical Co.) was added to a final concentration of $8 \mu \mathrm{g} / \mathrm{mL}$. After $48 \mathrm{~h}$ of incubation, the medium was replaced with fresh K562 growth medium, and the cells were incubated for an additional $24 \mathrm{~h}$. Transduced K562 cells were fixed by incubation in phosphate-buffered saline (PBS) containing 2\% formaldehyde (Polysciences, Inc.) for $30 \mathrm{~min}$ on ice and then permeabilized by incubation in 1\% Tween 20 (Fisher Scientific) for 45-60 min at room temperature. Expression of the fusion protein was detected by flow cytometry with a BD LSRI flow cytometer (Becton Dickinson) after immunolabeling with an anti-firefly luciferase monoclonal antibody (Novus Biologicals) and a phycoerythrinconjugated antimouse secondary antibody (Caltag Laboratories).

\section{Isolation and Transduction of hMSCs}

Bone marrow was obtained as scoops from patients undergoing hip or knee arthroplasty after patient consent and approval by the Institutional Review Board of the University Hospitals of Cleveland. The marrow sample was combined with $25 \mathrm{~mL}$ of DMEM containing a low concentration of glucose $(1 \mathrm{~g} / \mathrm{L})$ (DMEM-LG; Sigma Chemical Co.) and supplemented with $1 \%$ (v/v) antibioticantimycotic solution (Life Technologies), 1\% GlutaMAX, and 10\% hMSC-tested FBS (Hyclone Laboratories). The cell suspension was centrifuged at $500 \mathrm{~g}$ for $5 \mathrm{~min}$. After the supplement was removed, the pellet was resuspended in $5 \mathrm{~mL}$ of serum-supplemented DMEM-LG, and the suspension was carefully layered over $35 \mathrm{~mL}$ of $63 \%(\mathrm{v} / \mathrm{v})$ Percoll in Tyrode's salt solution (Sigma Chemical Co.) in a sterile, capped polycarbonate centrifuge tube, with the final sodium chloride concentration adjusted to $0.1 \mathrm{~mol} / \mathrm{L}$. The sample was centrifuged at $20,000 \mathrm{~g}$, and the top $25 \%$ of the gradient (pool density, $1.03 \mathrm{~g} / \mathrm{mL}$ ) was removed and transferred to a 50-mL polypropylene centrifuge tube (Becton Dickinson). Serumcontaining medium was added to a volume of $50 \mathrm{~mL}$, the tube was centrifuged at $500 \mathrm{~g}$, and the pellet was resuspended in $7 \mathrm{~mL}$ of serum-supplemented DMEM-LG. The number of nucleated cells in the suspension was determined by counting the cells in a $4 \%$ $(\mathrm{v} / \mathrm{v})$ acetic acid-treated sample with a hemacytometer. Nucleated cells were used to seed $100-\mathrm{mm}$ tissue culture dishes $\left(10^{7}\right.$ cells per dish). The medium was changed after $3 \mathrm{~d}$ and twice weekly thereafter. When cell colonies became large, the cells were subcultured by treatment with trypsin $(0.25 \%)$-ethylenediaminetetraacetic acid (EDTA; $1 \mathrm{mmol} / \mathrm{L}$ ) (Life Technologies) for $5 \mathrm{~min}$ at $37^{\circ} \mathrm{C}$. Trypsinization was arrested by the addition of a volume of calf serum equal to half the volume of trypsin-EDTA. The cell suspension was transferred to a $50-\mathrm{mL}$ polypropylene tube and centrifuged at $500 \mathrm{~g}$ for $5 \mathrm{~min}$. The cells were resuspended and counted with a hemacytometer, and $2 \times 10^{5}$ cells were used to seed 100 $\mathrm{mm}$ dishes containing serum-supplemented DMEM-LG. The medium was then changed after $3 \mathrm{~d}$ and twice weekly thereafter.

Primary hMSCs were transduced with virus obtained from either pHR'-mnd-Not transfections (yielding empty-vector-transduced hMSCs as one type of control) or $\mathrm{pHR}^{\prime}$-mnd-LRT transfections (yielding reporter-transduced hMSCs for imaging). The hMSCs were used to seed $175-\mathrm{cm}^{2}$ flasks containing hMSC growth medium and were grown to $30 \%$ confluence. The cells were incubated with virus at a multiplicity of infection of 8 in $25 \mathrm{~mL}$ of hMSC growth medium containing Polybrene at $8 \mu \mathrm{g} / \mathrm{mL}$; the transduction protocol was repeated one time for all hMSC cultures. In each round, cells were incubated with viral supernatant for $8 \mathrm{~h}$ and then in hMSC growth medium for $16 \mathrm{~h}$. After the second round, fresh medium was added to each flask, and the cells were incubated for an additional $24-48 \mathrm{~h}$. The cells were passaged $1: 4$ and grown to $80 \%-100 \%$ confluence. Three days after transduction, the cells were viewed on an Eclipse TE200 inverted microscope equipped with a fluorescence source and a chargecoupled device (CCD) camera. Transduction efficiency was determined by fluorescence-activated cell sorting (FACS) analysis of mrpf expression with the same setting as that previously described (20).

\section{Detection of Transduction and Expression}

Wild-type or nontransduced hMSCs (another type of control), empty-vector-transduced hMSCs, and reporter-transduced hMSCs were harvested $6 \mathrm{~d}$ after transduction. The cells were trypsinized for $5 \mathrm{~min}$ as described earlier and transferred to a $50-\mathrm{mL}$ polypropylene centrifuge tube. The cells were pelleted by centrifugation at $5,000 \mathrm{~g}$ for $5 \mathrm{~min}$ at $4^{\circ} \mathrm{C}$ (Sorvall RT 6000D centrifuge; Thermo Electron, Inc.). The supernatant was discarded, and the cells were rinsed by resuspension in PBS (Invitrogen). The cells were rinsed a total of 3 times. The cell pellets were homogenized in $500 \mu \mathrm{L}$ of lysis buffer, containing $1 \mathrm{mM}$ Tris- $\mathrm{HCl}(\mathrm{pH} \mathrm{7.0)}$, $3 \mathrm{mM}$ 2-mercaptoethanol, and 0.5\% Igepal CA-630 (SigmaAldrich), and centrifuged at $13,600 \mathrm{~g}$ for $20 \mathrm{~min}$ at $4{ }^{\circ} \mathrm{C}$ with the Sorvall RT 6000D centrifuge. The resulting supernatants were used for luciferase and HSV1 thymidine kinase enzyme assays.

Luminometer Assay for fluc. Luciferase enzyme activity was assayed by incubating $10 \mu \mathrm{L}$ of cell lysate supernatant with $80 \mu \mathrm{L}$ of D-luciferin (Promega Inc.). Luminescence was measured with a $20 / 20^{\text {n }}$ luminometer (Turner Biosystems, Inc.). The total protein content in the supernatant was determined by the Bradford (28) method (Bio-Rad Laboratories, Inc.). The activity was expressed in relative light units per milliliter of cell lysate and normalized to the total protein content. All samples were assayed in triplicate.

Thymidine Kinase Assay for tk. Lysates from wild-typetransduced, empty-vector-transduced, and reporter-transduced hMSCs were obtained as described earlier. The samples were assayed for thymidine kinase enzyme activity with $8-{ }^{3} \mathrm{H}$-ganciclovir (Moravek Biochemicals, Inc.) as described by Gambhir et al. (29). A 5- $\mu \mathrm{L}$ volume of cell lysate was incubated for 30 min with $3 \mu \mathrm{L}$ of thymidine kinase reaction buffer $\left(8-{ }^{3} \mathrm{H}\right.$-ganciclovir, $0.5 \mathrm{M}$ dibasic anhydrous sodium phosphate [pH 6.0], 0.1 M adenosine triphosphate [pH 7.0], and $1 \mathrm{M}$ magnesium acetate) to determine the formation of phosphorylated $8-{ }^{3} \mathrm{H}$-ganciclovir. After termination of the reaction, $8{ }^{-3} \mathrm{H}$-ganciclovir was separated from phosphorylated $8-{ }^{3} \mathrm{H}$-ganciclovir with a $2.3-\mathrm{cm}$ DE-81 filter (Whatman) (30). The radioactivity of phosphorylated $8{ }^{3} \mathrm{H}$-ganciclovir was measured with a Beckman LS-6500 liquid scintillation counter (Beckman Coulter) and Bio-safe II scintillation fluid (RPI, Inc.). Disintegrations per minute (dpm) were obtained by correcting for background activity and efficiency (61\% for tritium) based on calibrated standards (Beckman Coulter). Tritium counts were also corrected for quenching effects. The total protein content in the supernatant was determined by the Bradford (28) method (BioRad Laboratories, Inc.). The activity was expressed in $\mathrm{dpm} / \mu \mathrm{g}$ of protein/min. All samples were assayed in triplicate.

\section{In Vitro Characterization of Transduced hMSCs}

Primary and first-passage hMSCs transduced with the imaging reporter were tested for transgene expression as described earlier and were assayed for the potential to differentiate into osteogenic, chondrogenic, and adipogenic lineages as described later. The differentiation potential of transduced hMSCs was directly compared 
with that of wild-type hMSCs obtained from the same donor to determine whether lentiviral transduction of the reporter system altered or interfered with the normal functions of the hMSCs.

Osteogenic Potential Assay. A total of $5.0 \times 10^{4} \mathrm{hMSCs}$ at $30 \%$ confluence were used to seed 12 -well tissue culture plates $(3.0 \times$ $10^{3}$ cells per square centimeter) and allowed to attach overnight. On the next day (day 0), the culture medium was completely removed, and the tissue culture plates were divided into 2 groups; one group contained growth medium (DMEM with $10 \%$ FBS, as a negative control for osteogenesis), and the other group contained osteogenic medium (which was composed of growth medium with $10^{-7} \mathrm{M}$ dexamethasone [Dex], $2 \mathrm{mM} \beta$-glycerophosphate, and $0.05 \mathrm{mM}$ ascorbic acid 2-phosphate [Wako]). Complete medium changes were performed twice weekly, with a medium volume of $2 \mathrm{~mL}$ per well. Cultures were harvested on days 10, 20, and 30 . Calcium deposited into the cell layer was extracted with $0.5 \mathrm{~N} \mathrm{HCl}$ and then quantified with a commercial kit (Bi-Tron) according to the manufacturer's instructions. The absorbance was read at $575 \mathrm{~nm}$ on a microplate reader (Tecan Systems, Inc.). The calcium concentration was calculated from standard solutions prepared in parallel and expressed as micrograms per well. The mean level of calcium deposition at each time was calculated, and the levels were compared between transduced and wild-type hMSCs.

Chondrogenic Potential Assay. After being washed with serumfree DMEM-LG, hMSCs were divided into aliquots of $2.5 \times 10^{5}$ cells in $0.5 \mathrm{~mL}$ of defined medium (DMEM with a high glucose concentration and with ITS + Premix [Collaborative Biomedical Products]) with pyruvate (1 $\mathrm{mM}$; Gibco BRL), ascorbic acid 2-phosphate $(100 \mu \mathrm{M})$, Dex $\left(10^{-7} \mathrm{M}\right)$, and recombinant human transforming growth factor- $\beta 1$ (10 ng/mL; Peprotech) in a $15-\mathrm{mL}$ conical polypropylene centrifuge tube. The cells were pelleted by centrifugation at $500 \mathrm{~g}$ for $5 \mathrm{~min}$. The aggregates were incubated at $37^{\circ} \mathrm{C}$ in $95 \%$ humidified air and $5 \% \mathrm{CO}_{2}$. The culture medium was changed every other day. Twenty-four hours after seeding, the aggregates were tapped briefly to detach them from the bottom of the tube. For histologic analysis, aggregates were fixed for $15 \mathrm{~min}$ in $10 \%$ neutral buffered formalin, dehydrated in a series of graded alcohols, embedded in paraffin, and sectioned at a thickness of $5 \mu \mathrm{m}$. For immunostaining, sections were incubated with an antitype II collagen antibody (II-II6B3; purchased by Dr. Thomas Linsenmayer from the Developmental Studies Hybridoma Bank) diluted 1:100 in $1 \%$ bovine serum albumin (BSA) in PBS; the sections were washed, incubated with a biotinylated goat antimouse antibody (Cappel; Organon Teknika) diluted 1:100 in 1\% BSA, washed, and incubated with streptavidin-peroxidase diluted 1:300 in $1 \%$ BSA in PBS. The slides were washed with PBS, treated with a VIP Substrate Kit (Vector Laboratories) according to the manufacturer's instructions, washed, and counterstained with $1 \%$ Fast Green.

Adipogenic Potential Assay. Second-passage hMSCs were plated in adipogenic medium (growth medium with insulin [5 mg/mL], $10^{-6} \mathrm{M}$ Dex, $0.5 \mathrm{mM}$ isobutylmethylxanthine, and $60 \mathrm{mM}$ indomethacin [made from a 1,000-fold stock in dimethyl sulfoxide]). At 8 and $14 \mathrm{~d}$ of culturing, cells were released from tissue culture plates by trypsin digestion, fixed by the addition of $4 \%$ paraformaldehyde to a final concentration of $0.5 \%$, and stained for triglycerides with a solution of Nile Red in PBS $(1 \mathrm{mg} / \mathrm{mL})$. The intensity of staining within the cells was measured at the Flow Cytometry Core Facility at Case Western Reserve University (Cancer Center Core Facility). Cells cultured in growth medium served as baseline controls for measuring Nile Red staining, and adipogenic cells were defined as cells having a staining intensity equal to or greater than that of the upper $1 \%$ of the growth medium sample.

\section{Mouse Models}

NOD-SCID mice were used as recipients for all hMSC transplants or implants. This animal model, which is deficient in B, T, and natural killer cells, does not reject human cells and has been successfully used to test the engraftment of human hematopoietic cells (31). All animals were housed and cared for in the Case Western Reserve University Animal Resource Center. All animal procedures were implemented according to approved Institution Animal Care and Use Committee protocols. The NOD-SCID mice in this study were $8 \mathrm{wk}$ old at the time of the experiments. Reporter-transduced hMSCs (for imaging) were loaded into porous, fibronectin-coated ceramic cubes (Zimmer, Inc.), which were subcutaneously implanted into NOD-SCID mice. It was previously determined that about 50,000 cells can be loaded into a cube by this procedure (32). Cubes were loaded with either reportertransduced hMSCs or a 1:4 mixture of reporter-transduced hMSCs and empty-vector-transduced hMSCs (to assay for a quantitative measurement based on image intensity). Control cubes were loaded with wild-type hMSCs, empty-vector-transduced hMSCs, or no hMSCs (empty cubes, yet another control). The mice were divided into 2 groups: one $(n=5)$ loaded with 6 cubes and the other $(n=$ 4) loaded with 8 cubes. This grouping strategy allowed for intrasubject positive and negative controls. Generally, a pair of identical cubes were inserted at each longitudinal position. For example, for mice receiving 8 cube implants, 2 cubes were loaded with reporter-transduced hMSCs, 2 were loaded with the 1:4 mixture, 2 were loaded with wild-type hMSCs, and 2 were empty cubes. For mice receiving 6 cube implants, 2 cubes were loaded with reporter-transduced hMSCs, 2 were loaded with wild-type hMSCs, and 2 were empty cubes.

\section{BLI}

After receiving hMSCs via cube implants, mice were imaged by BLI at $30 \mathrm{~min}, 24 \mathrm{~h}, 96 \mathrm{~h}$, and once per week after that for up to 3 mo. Each mouse was anesthetized with isoflurane administrated by use of an EZ-Anesthesia system (Euthanex) and was restrained in a horizontal position after induction. At 5-6 min before each scan, each animal was given an intraperitoneal injection of $0.2 \mathrm{mg}$ of D-luciferin in $0.2 \mathrm{~mL}$ of sterile PBS. Imaging was performed with either a Xenogen IVIS 200 System (Xenogen) or a PIXIS 1000 System (Princeton Instrument) cooled CCD camera. Exposure times were 3 and $5 \mathrm{~min}$, respectively. Although still under anesthesia, the mice were placed on a special planar $\mathrm{x}$-ray system developed jointly by Thomas Jefferson National Laboratory and Case Western Reserve University to precisely determine cube positions; x-ray exposure was limited to $6 \mathrm{~s}$. Digital data acquisition allowed a spatial resolution of about $50 \mu \mathrm{m}$ on the radiographs. In order to correlate the BLI signal with the cube location, BLI and planar x-ray images were aligned by use of the Pmod (Pmod Technologies) software package, which allowed semiquantitative analysis of BLI data in defined regions of the radiographs.

\section{CT and Small-Animal PET Imaging}

After BLI, 1 or 2 mice with brighter BLI signals were selected for imaging by CT and PET at $96 \mathrm{~h}, 1 \mathrm{mo}$, and 2 mo after implantation. Each selected mouse was anesthetized with isoflurane as described earlier. Animals were scanned by small-animal CT with the CT component of an X-SPECT scanner (Gamma Medica). After the CT scan, the animals were moved to an R4 
microPET scanner (Siemens Medical Solutions) for a 20-min transmission scan with the point source. A dose of $9.25 \mathrm{MBq}$ of 9-[4- ${ }^{18} \mathrm{~F}$-fluoro-3-(hydroxymethyl)butyl]guanine $\left({ }^{18} \mathrm{~F}-\mathrm{FHBG}\right)$ in $0.2 \mathrm{~mL}$ of sterile saline was injected intravenously via the tail vein. At $20 \mathrm{~min}$ after injection, a 40-min dynamic PET emission scan was obtained. The radiotracer ${ }^{18} \mathrm{~F}$-FHBG was synthesized as described by Alauddin and Conti (33).

\section{Image Data Analysis}

The PET images were reconstructed by use of a 2-dimensional ordered-subset expectation maximization algorithm with a voxel size of $0.845 \times 0.845 \times 1.211 \mathrm{~mm}$. Alignment between PET emission and CT images was done by registering the CT images with the PET transmissions by use of software developed inhouse. Various regions of interest (ROIs) were centered on cubes seen on the CT images that were aligned with the PET images binned for the last $10 \mathrm{~min}$. Regional data were defined as the sum of the measured radioactivities within a given region at a specific time point. For each ROI, data were decay corrected. Relative uptake was calculated as the ratio of ROI-based uptake values between cubes containing different amounts of transduced hMSCs.

\section{Histologic Analysis for In Vivo Osteogenesis}

Selected mice were euthanized at selected time points after BLI and PET by an overdose of anesthesia, and cubes corresponding to the ROIs on the images, as mentioned earlier, were harvested. The ceramic cubes removed from the animals were placed immediately into $10 \%$ formalin in PBS, fixed for $24 \mathrm{~h}$, washed with water, decalcified in routine acid decalcification (Apex Engineering), and prepared for standard histologic analysis. Serial sections, cut at $5 \mu \mathrm{m}$, were stained with Mallory-Heidenhain stain and examined for bone formation.

\section{RESULTS}

\section{hMSC Transduction}

The mean titer of lentivirus produced by transient cotransfection was $8 \times 10^{5}$ infectious particles per milliliter. Tranduction of hMSCs at a multiplicity of infection of 8 yielded a transduction efficiency of $83.2 \%$, as determined by $m r f p$ FACS analysis (Fig. 1A).

\section{In Vitro Functional Assays}

For transduced hMSCs, densities of passaged cells were equal to that of wild-type hMSCs, indicating comparable growth rates (data not shown). Aliquots of transduced cells were assayed in vitro for $f l u c$ and $t k$ activities by luminometer and radiolabel assays, respectively. Reporter-transduced hMSCs displayed in vitro luciferase activity 4,000-fold greater than that of wild-type hMSCs. Reporter-transduced hMSCs had $t k$ activity 9-fold greater than that of wild-type hMSCs. Reporter-transduced hMSCs were visible by fluorescence microscopy (Fig. 1B) beginning $3 \mathrm{~d}$ after transduction.

\section{In Vitro Characterization of Pluripotential Capacity}

Reporter-transduced hMSCs were compared with wildtype and empty-vector-transduced hMSCs in terms of their ability to differentiate into adipogenic, chondrogenic, and osteogenic lineages.

Stem cells were induced to form adipocytes as described earlier for adipogenic potential assays and were assessed for the proportion of cells staining with Nile Red, a measure of cellular lipophilic content. As determined with this method, $57 \% \pm 3.6 \%$ (mean $\pm \mathrm{SD}$ ) of reporter-transduced hMSCs had an adipocytic phenotype; the corresponding values for empty-vector-transduced and wild-type hMSCs were $34 \% \pm 6.4 \%$ and $31 \% \pm 0.9 \%$, respectively (Fig. 2A).

Reporter-transduced, empty-vector-transduced, and wildtype hMSCs were incubated in osteogenic medium for the osteogenic potential assay. As controls, cell samples were also incubated without Dex. Calcium was extracted and measured as an indicator of osteoblastic differentiation. The results shown in Figure 2B indicated that the osteogenic potential of transduced hMSCs was preserved.

For the chondrogenic potential assay, all hMSCs were induced to form chondrogenic cell pellets in medium containing insulin, transferrin, selenious acid, dexamethasone, sodium pyruvate, ascorbic acid 2-phosphate, and transforming growth factor- $\beta 1$. The resulting pellets were fixed and stained for type II collagen (purple staining; the negative areas showing light green around the edge were attributable to counterstaining) for a visual assessment of chondrogenic potential. As shown in Figure 2C, there was no obvious difference between the transduced hMSCs and the wild-type hMSCs.

\section{Images of Cube Implants}

The cubes loaded with reporter-transduced hMSCs were visualized by BLI $30 \mathrm{~min}$ after implantation. Figure 3A shows the BLI signal $4 \mathrm{~d}$ after cube implantation. The

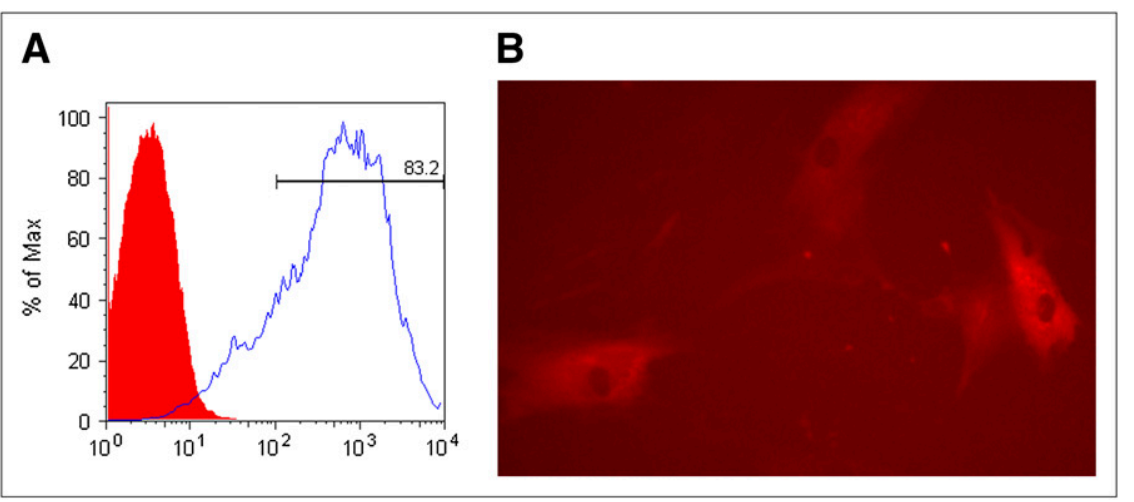

FIGURE 1. Lentiviral transduction of triple-fusion reporter. (A) mrfp FACS scans with Texas Red $(585 \pm 25 \mathrm{~nm})$ filter setting. Red peak is from control cells. (B) Red-fluorescent adherent transduced hMSCs viewed $3 \mathrm{~d}$ after transduction at $20 \times$ magnification with Nikon Eclipse TE200 inverted microscope equipped with CCD camera. Max = maximum intensity. 
FIGURE 2. Differentiation potentials of reporter-transduced, empty-vector transduced, and wild-type (nontransduced) hMSCs. (A) Adipogenesis measured by flow cytometry after Nile Red staining, expressed as percentage above values for control samples from day 1. (B) Elevated calcium levels in all transduced and wild-type samples vs. internal control samples not treated with osteogenic medium (labeled as "control" in both $A$ and B). (C) Type II collagen-stained histologic sections showing metachromasia (glycosaminoglycans) in wild-type hMSCs, reporter-transduced hMSCs, and empty-vector-transduced hMSCs.

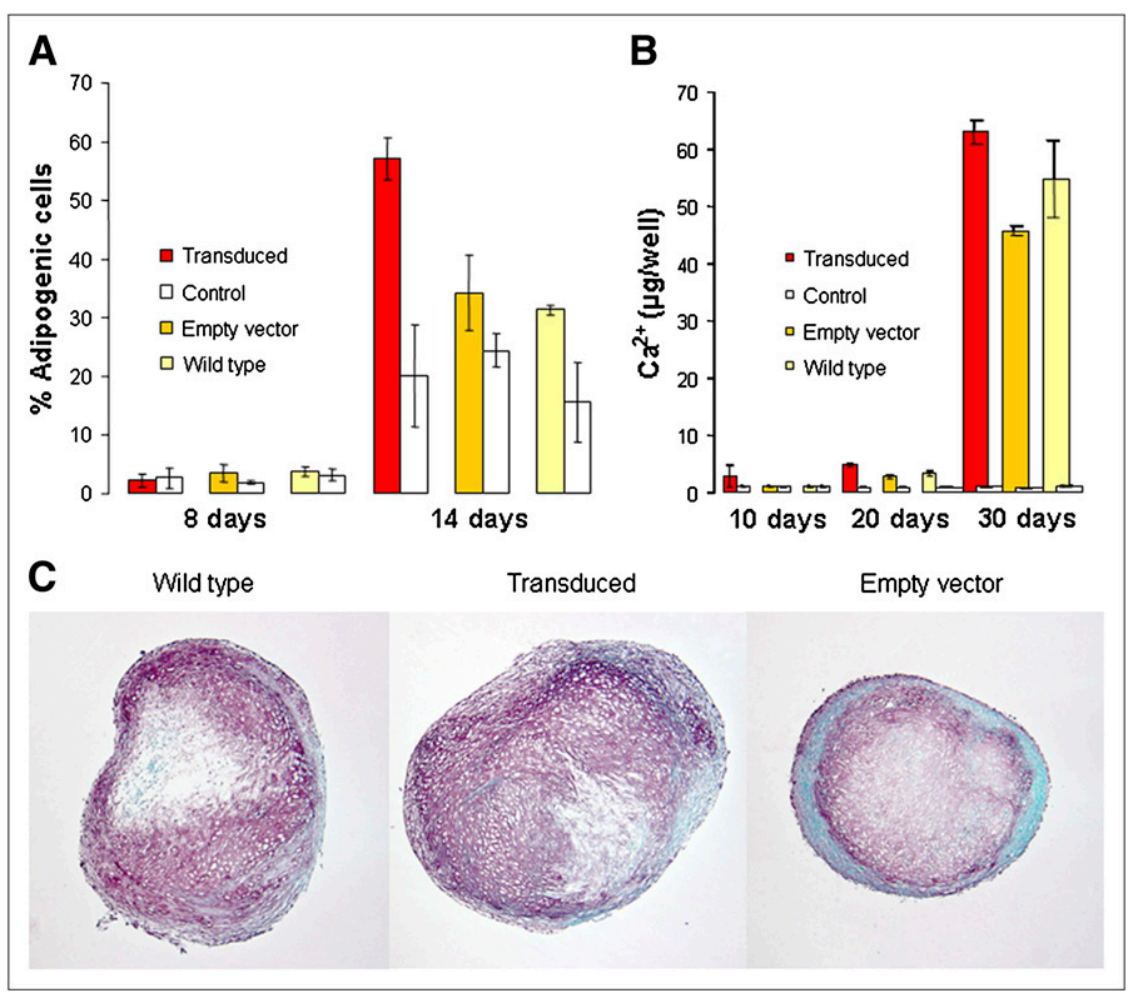

staples remained for the surgical wound created during implantation. Compared with the wild-type hMSC-loaded or empty cubes in the same animal, only the cubes loaded with reporter-transduced hMSCs produced an imaging signal. Figure $3 \mathrm{~B}$ shows an image from the same animal as that in Figure 3A but with the BLI image superimposed on the radiograph, demonstrating the origin of the signal

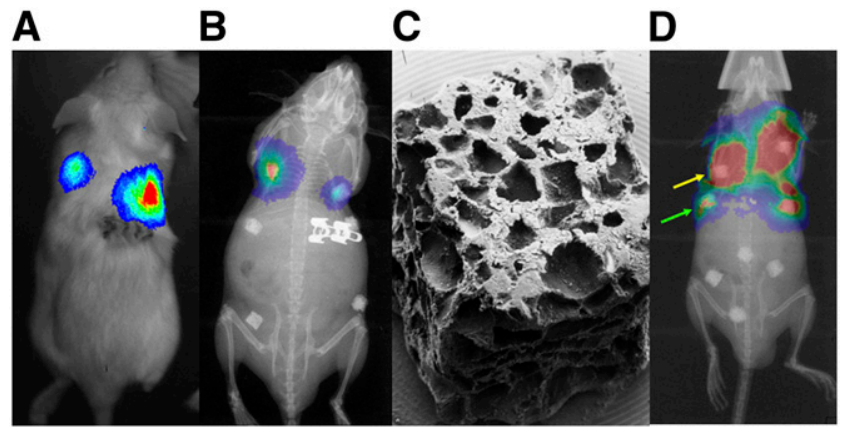

FIGURE 3. BLI of cube implants. (A and B) BLI overlaid on photograph $(A)$ and $B L I$ overlaid on radiograph $(B)$. Both mice had 6 cubes: top 2 loaded with reporter-transduced hMSCs, middle 2 loaded with wild-type hMSCs, and bottom 2 loaded with empty-vector-transduced hMSCs located at caudal midline, and empty ceramic cubes located caudally and laterally. (C) Scanning electron micrograph of representative ceramic cube for cell loading and implantation. (D) BLI overlaid on radiograph in animal implanted with reporter-transduced hMSCs (top row, yellow arrow), mixture of empty-vectortransduced and reporter-transduced hMSCs (second row, green arrow), empty-vector-transduced hMSCs located at midline, and empty ceramic cube located laterally. from the implanted ceramic cubes. Again, the metal staples were clearly visible. The images in both Figures 3A and 3B were taken with the PIXIS camera at the Athymic Animal Facility of the Animal Resources Center of Case Western Reserve University. The size of a typical porous ceramic cube used for loading of the cells was $0.3 \times 0.3 \times 0.3 \mathrm{~cm}$ (or $0.027 \mathrm{~cm}^{3}$ ), as shown in Figure 3C. Figure 3D shows the BLI image overlaid on the planar radiograph for a mouse with an 8-cube implant; the image was obtained with the Xenogen camera $4 \mathrm{~d}$ after implantation. There was a remarkable difference between the diluted and undiluted cells transduced with the triple-fusion reporter. For the upper 2 cubes, loaded with undiluted hMSCs transduced with the reporter, a larger region showing a signal resulted from the massive diffusion and scattering of the visiblelight photons. The BLI signal was detectable beyond 3 mo after cube implantation, during which time the BLI signal intensity from the loaded cubes went up and down. No animals were kept longer than 14 wk after implantation, and animals were euthanized for histologic examination.

Selected animals from BLI studies underwent smallanimal PET imaging at selected time points. Figure 4 shows an overlay of PET and CT images for a mouse with an 8 -cube implant $4 \mathrm{~d}$ after cube implantation. The upper 2 cubes, loaded with undiluted stem cells, were clearly visible, and even the cubes loaded with diluted cells were visible on the small-animal PET scan. Quantitative results from ROIs defined around these cubes showed that the signal intensity of the upper (undiluted) cubes was, on average, $2.63 \pm 0.52$ times higher than that of the second-row (diluted) cubes (the 


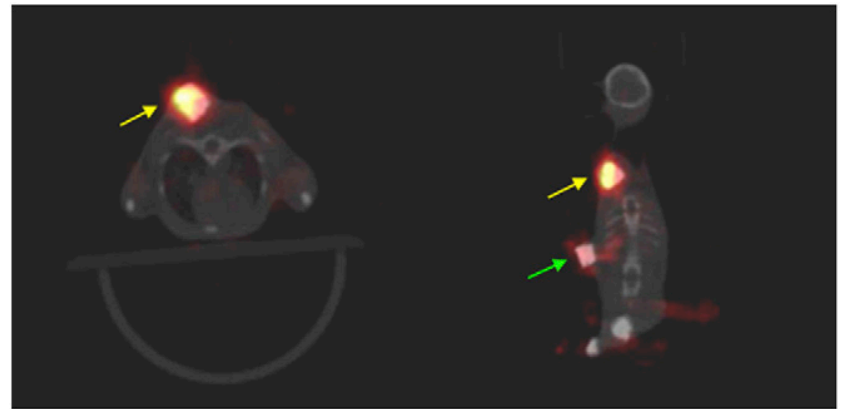

FIGURE 4. Overlay of PET (hot-metal pseudocolor) and CT (gray-scale) images: transaxial (left) and coronal (right) views. Although top-row cubes (yellow arrows) had strong PET signals, second-row cubes (green arrow), loaded with mixture of empty-vector-transduced and reporter-transduced hMSCs, had visible signal despite 1:4 dilution. Cubes were also visible on CT images.

ratio ranged from 3.27 to 2.05); the concentration ratio of reporter-transduced hMSCs was 4:1 in the cell preparations before loading into their corresponding cubes. The PET signal from the cubes remained detectable for $2.5 \mathrm{mo}$, when the last small-animal PET scan was performed.

\section{Histologic Analysis}

The osteogenic potential of transduced and wild-type hMSCs was assessed by histologic examination. Although hMSCs transduced with an empty vector and with the triple-fusion reporter showed robust bone formation (Fig. $5 \mathrm{~A})$, empty ceramic cubes showed either no bone formation or, at most, only trace amounts of bone in a few ceramic pores (Fig. 5B). There was no discernible difference between empty-vector-transduced hMSCs and reportertransduced hMSCs.

\section{DISCUSSION}

\section{Transduction of Triple-Fusion Reporter into hMSCs}

The efficiency of lentiviral transduction of the triplefusion reporter into hMSCs was found to be $83.2 \%$, a value that is higher than that obtained with a similar lentiviral vector and mouse stem cells (19). Enzymatic assays and a fluorescence examination showed that all 3 components of the triple-fusion reporter were working properly. The other assay results showed that reporter-transduced hMSCs had a normal growth pattern when compared with wild-type hMSCs. More importantly, it appeared that the phenotype and pluripotentiality of stem cells were retained after reporter transduction by use of the lentiviral vector, as demonstrated by adipogenic, osteogenic, and chondrogenic potential assays performed for both transduced and wildtype cells both in vitro and in vivo. With regard to other possible effects of such transduction on stem cells, a recent study indicated that mouse embryonic stem cells transduced with a similar lentiviral vector containing the same triple-fusion reporter did not show any significantly altered gene expression profiles (23). Our own DNA microarray

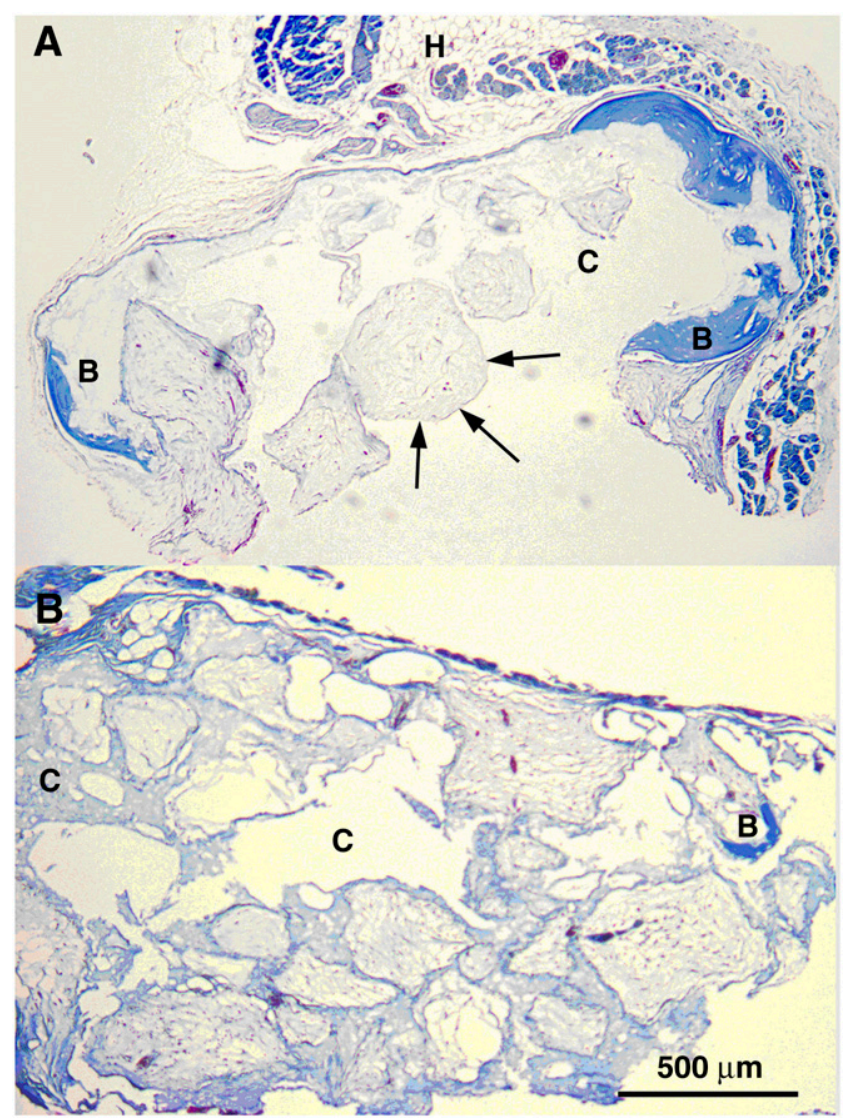

FIGURE 5. (A) Histologic section of ceramic cube loaded with transduced hMSCs and harvested $6 \mathrm{wk}$ after implantation into SCID/NOD mice, showing extensive areas of bone formation. (B) Section of control empty ceramic cube, showing only sliver of bone formation. Most sections were completely negative. $\mathrm{B}=$ bone; $\mathrm{C}=$ space occupied by ceramic cube before demineralization; $\mathrm{H}=$ host tissue; arrows indicate edge of ceramic pore.

studies (to be published elsewhere) performed to examine any transcriptional changes attributable to the transduction of the reporter by use of the lentiviral vector into hMSCs were just completed, and they did not show upregulation of any genes or transcripts that favor adipogenesis or osteogenesis. Although increased adipogenesis was noted for reporter-transduced cells, these transduced cells did not show any significantly different behavior in terms of transcriptional profile, growth rate, and differentiation potentials when compared with empty-vector-transduced and wild-type cells. Further investigation is needed to explain the increased adipogenesis.

\section{Feasibility of Long-Term Monitoring}

A modified myeloid proliferative sarcoma virus promoter (mnd promoter) was used to drive the expression of the triple-fusion reporter. This strong promoter drives the continuous expression of the triple-fusion reporter, allowing us to image implanted stem cells for more than 3 mo. We euthanized the animals for histologic evaluation of new bone formation, and the signal intensity from the images at 
that time point remained strong, suggesting the possibility of a longer duration of reporter gene expression had we kept the animals for repeated imaging. A previous study with a cytomegalovirus (CMV) promoter (34) and our own experience with CMV for reporter gene imaging (unpublished data) resulted in the silencing of the CMV promoter in stem cells and the loss of the imaging signal at certain time points after implantation. The choice of mnd as the promoter clearly prevented such epigenetic effects. More BLI images were collected along most preset time points, and only a few small-animal PET imaging studies were performed. This is because BLI studies are relatively easy to conduct and require no radiotracer and far less effort in image processing and data analysis after imaging compared with small-animal PET or small-animal CT imaging. When the BLI data were examined retrospectively, it was found that the imaging signals went up and down among different animals and among different cubes in the same animal. Because of the qualitative nature of the BLI technique, it is difficult to determine whether there is a "cycle" for an animal or for the cells in a given cube.

\section{Assessment with Small-Animal PET Image Data}

Using the cube implants allowed us to test the sensitivity of small-animal PET imaging because a known number of cells expressing the reporter were loaded into a cube for implantation. For a typical porous ceramic cube that we used, the volume occupancy in the body was $0.027 \mathrm{~cm}^{3}$, and previous work showed that approximately 50,000 hMSCs adhere to fibronectin-coated cubes (32). The transduction efficiency was $83.2 \%$; therefore, the number of transduced cells in a cube was about 41,600. These data led to a density of reporter-transduced hMSCs in a cube of slightly more than $1.54 \times 10^{7} / \mathrm{mL}$. For the cubes that were diluted with cells without the reporter, the portion of transduced hMSCs was only one-fourth that in the undiluted cubes. These data led to a density of transduced cells for imaging of approximately $385,000 / \mathrm{mL}$. The absolute number of transduced hMSCs producing detectable PET signals in the diluted cubes was estimated to be about 10,000. Whether this value is the absolute lower limit for PET detection remains to be seen. Previously published data, mostly from studies with tumor cells, on the sensitivity of the same type of small-animal PET scanner in detecting thymidine kinase-expressing cells with the same tracer $\left({ }^{18} \mathrm{~F}-\mathrm{FHBG}\right)$ revealed a lower threshold of smallanimal PET detection at a cell density of about $1.0 \times 10^{7}$ / $\mathrm{mL}(19,35)$. The undiluted cubes had a cell density that was higher than this lower threshold of small-animal PET detection, but cell density in the diluted cubes that were detectable on small-animal PET imaging was below the lower threshold. We speculate that this result may have been attributable to the fact that we used a much stronger promoter (mnd). In addition, human MSCs are larger than other cell types, such as tumor cells. The transduced hMSC concentration was planned to be 4 times higher in the undiluted cubes than in the diluted ones before cells were prepared and loaded into the cubes. Ideally, the ratio of reporter-transduced cells in the undiluted versus the diluted cubes would be 4:1. However, small-animal PET image intensities $4 \mathrm{~d}$ after implantation revealed differences of only 3.27 - to 2.05 -fold between undiluted and diluted cubes. Such differences can be explained by possible variations during the initial cell loading or changes in response to the in vivo microenvironment during the first $4 \mathrm{~d}$ after implantation. It is more likely that the spillover of high levels of uptake of ${ }^{18} \mathrm{~F}$-FHBG in the gut (because of the lipophilicity of ${ }^{18} \mathrm{~F}-\mathrm{FHBG}$ ) into the diluted cubes nearby artificially altered the counts in those cubes.

\section{Transplant Perspectives}

We designed these experiments with known amounts of reporter-transduced cells in each implanted cube to establish the feasibility of imaging at various concentrations of transduced stem cells in vivo in terms of the signal-tobackground ratio; this method served as a standard loading method and can be a positive control for imaging in future systemic delivery experiments. In a clinical setting, cube loading or implantation, intravenous injection, and sitespecific direct injection of hMSCs are possible means for hMSC transplantation. Only cube loading or implantation was studied extensively with the small-animal model in the present study. We did start experimenting with tail vein injection and BLI at $30 \mathrm{~min}$ after cell infusion; the preliminary results are shown in Figure 6A. The animal injected with wild-type hMSCs showed no signal, whereas the animals injected with reporter-transduced hMSCs showed intense signals emanating from the region of the lungs, indicating pulmonary entrapment of infused hMSCs. The images from $24 \mathrm{~h}$ after injection showed weakened signals emanating from the lungs, indicating dispersal of the hMSCs from the lungs (Fig. 6B). The initial entrapment in the lungs may have been attributable to their large size, which allowed gradual dispersal from the lungs in 1 or $2 \mathrm{~d}$. Pulmonary entrapment and subsequent release are common phenomena in nuclear medicine $(36,37)$. Because the NODSCID mice that we used had no injuries, we could not image their engraftment to any site for repair work. Future work will entail repair models, such as a bone fracture, cartilage damage, or even acute myocardial infarction. Because interactions between MSCs and hematopoietic stem cells play an important role in the therapeutic effects of MSCs, we will also investigate the dynamics of the 2 types of stem cells through cotransplantation of MSCs and hematopoietic stem cells to study improved human hematopoietic cell engraftment in mouse models.

\section{Logistic Considerations}

BLI is sensitive, as indicated by its detection of cubes with transduced MSCs that were no longer detected by PET (at 3 mo after implantation). BLI can be performed several times per day, but perhaps such a schedule is not necessary, 


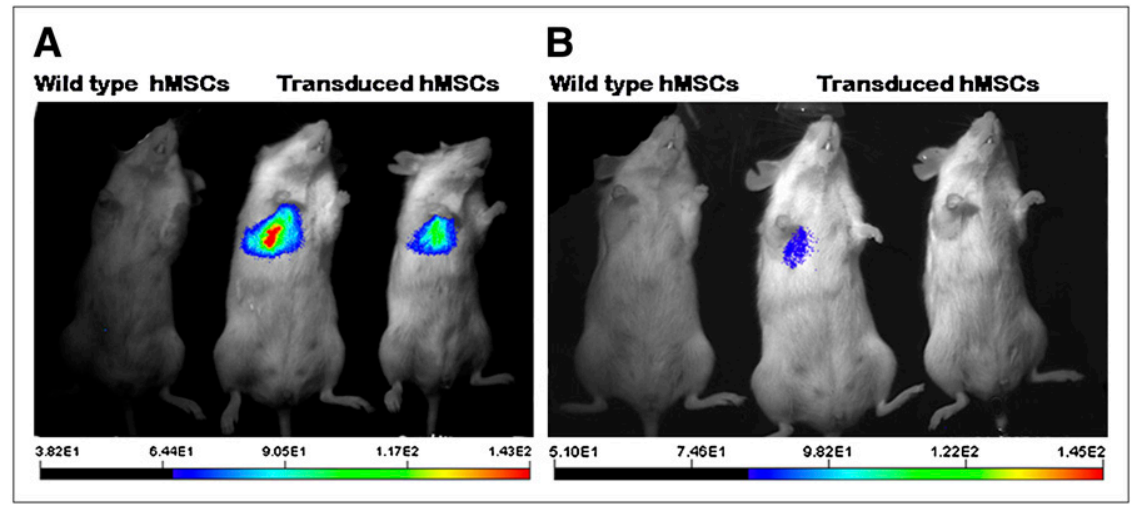

FIGURE 6. BLI of tail vein-injected hMSCs 30 min after hMSC injection (A) and $24 \mathrm{~h}$ after hMSC transplantation (B). One control animal injected with wildtype hMSCs is shown on left, and 2 animals injected with reporter-transduced hMSCs are shown on right. Scale bar for luminescence intensity is shown at bottom.

because the time frame for homing and engraftment of stem cells as well as their proliferation and differentiation is on the scale of days to weeks. PET can be performed once daily, allowing 10 half-lives of decay for an ${ }^{18} \mathrm{~F}-\mathrm{FHBG}$ tracer injection. However, because of the aseptic requirements for housing immunodeficient animals, such as the NOD-SCID mice used in the present study, one "clean" camera, the PIXIS cooled CCD camera, was placed in the Athymic Animal Facility permanently for repeated imaging. Only selected animals were taken out of the Athymic Animal Facility for small-animal PET imaging along with same-day BLI with the more sensitive Xenogen cooled CCD camera, and the animals were euthanized to harvest the cubes for histologic processing. This is why smallanimal PET imaging was performed far less frequently than BLI for the animals, along with the complexity of PET. The reporter gene strategy that we adopted is a "beacon indicator" that is on all the time for tracking of stem cells in vivo. However, when stem cells differentiate or exercise their functions, the imaging method described here cannot measure those activities. In the future, we will explore ways to introduce an additional gene(s) into triple-fusion reporter-positive stem cells to further image or measure functional events of the infused stem cells.

\section{CONCLUSION}

An understanding of the dynamics of homing and engraftment of infused stem cells is crucial for the progression of stem cell therapeutics. Noninvasive imaging in real time and in repeated fashion, as proposed here, is an ideal method for tracking stem cell transplantation. The specific triple-fusion reporter approach that we adopted resulted in a reliable method for labeling stem cells for investigation in small-animal models by use of both BLI and small-animal PET imaging and for potential translation into future human studies with clinical PET.

\section{ACKNOWLEDGMENTS}

We would like to thank Lisa Walsh for help with the ceramic cube implantation and Joseph Molter for BLI and for help with animal handling. In addition, our thanks go to Nell Ginley and Dr. In Hwan Song for technical assistance with the differentiation assays. We also thank Dr. Luis Solchaga for supplying hMSCs. We thank the Flow Cytometry Core Facility at Case Western Reserve University, under the direction of Dr. James Jacobberger and supported by NIH/NCI Grant CA43703, for their general support and for facilitating the connection with the Pittsburgh Flow Cytometry Core Facility. Finally, we thank the Flow Cytometry Facility at the University of Pittsburgh School of Medicine, under the direction of Dr. Albert Donnenberg, for help with mrfp FACS analysis. This work was supported in part by the DOE (DE-FG02-03ER63597); by NIH R21 (EB001847), NIH R24 (CA110943), NIH R01 (AR4978501A1), and NIH R01 (CA073062); and by JLab Subcontract SURA-04-Q011.

\section{REFERENCES}

1. Caplan AI. Mesenchymal stem cells. J Orthop Res. 1991;9:641-650.

2. Devine SM, Cobbs C, Jennings M, Bartholomew A, Hoffman R. Mesenchymal stem cells distribute to a wide range of tissues following systemic infusion into nonhuman primates. Blood. 2003;101:2999-3001.

3. Pittenger MF, Mackay AM, Beck SC, et al. Multilineage potential of adult human mesenchymal stem cells. Science. 1999;284:143-147.

4. Koc ON, Gerson SL, Cooper BW, et al. Rapid hematopoietic recovery after coinfusion of autologous-blood stem cells and culture-expanded marrow mesenchymal stem cells in advanced breast cancer patients receiving high-dose chemotherapy. J Clin Oncol. 2000;18:307-316.

5. Lazarus HM, Koc ON, Devine SM, et al. Cotransplantation of HLA-identical sibling culture-expanded mesenchymal stem cells and hematopoietic stem cells in hematologic malignancy patients. Biol Blood Marrow Transplant. 2005;11: 389-398.

6. Le Blanc K, Rasmusson I, Sundberg B, et al. Treatment of severe acute graftversus-host disease with third party haploidentical mesenchymal stem cells. Lancet. 2004;363:1439-1441.

7. Nakamizo A, Marini F, Amano T, et al. Human bone marrow-derived mesenchymal stem cells in the treatment of gliomas. Cancer Res. 2005;65:3307-3318.

8. Daley GQ, Goodell MA, Snyder EY. Realistic prospects for stem cell therapeutics. Hematology Am Soc Hematol Educ Program. 2003:398-418.

9. Li Y, Chen J, Chen XG, et al. Human marrow stromal cell therapy for stroke in rat: neurotrophins and functional recovery. Neurology. 2002;59:514-523.

10. Bulte JW, Arbab AS, Douglas T, Frank JA. Preparation of magnetically labeled cells for cell tracking by magnetic resonance imaging. Methods Enzymol. 2004;386:275-299.

11. Arbab AS, Yocum GT, Kalish H, et al. Efficient magnetic cell labeling with protamine sulfate complexed to ferumoxides for cellular MRI. Blood. 2004; 104:1217-1223.

12. Ma B, Hankenson KD, Dennis JE, Caplan AI, Goldstein SA, Kilbourn MR. A simple method for stem cell labeling with fluorine 18. Nucl Med Biol. 2005;32:701-705. 
13. Hofmann M, Wollert KC, Meyer GP, et al. Monitoring of bone marrow cell homing into the infarcted human myocardium. Circulation. 2005;111:2198-2202.

14. Gao J, Dennis JE, Muzic RF, Lundberg M, Caplan AI. The dynamic in vivo distribution of bone marrow-derived mesenchymal stem cells after infusion. Cells Tissues Organs. 2001;169:12-20.

15. Bindslev L, Haack-Sørensen M, Bisgaard K, et al. Labelling of human mesenchymal stem cells with indium-111 for SPECT imaging: effect on cell proliferation and differentiation. Eur J Nucl Med Mol Imaging. 2006;33: 1171-1177.

16. Jin Y, Kong H, Stodilka RZ, et al. Determining the minimum number of detectable cardiac-transplanted ${ }^{111}$ In-tropolone-labelled bone-marrow-derived mesenchymal stem cells by SPECT. Phys Med Biol. 2005;50:4445-4455.

17. Min JJ, Ahn Y, Moon S, et al. In vivo bioluminescence imaging of cord blood derived mesenchymal stem cell transplantation into rat myocardium. Ann Nucl Med. 2006;20:165-170.

18. Pelled G, Tai K, Sheyn D, et al. Structural and nanoindentation studies of stem cell-based tissue-engineered bone. J Biomech. 2007;40:399-411.

19. Hung SC, Deng WP, Yang WK, et al. Mesenchymal stem cell targeting of microscopic tumors and tumor stroma development monitored by noninvasive in vivo positron emission tomography imaging. Clin Cancer Res. 2005;11: 7749-7756.

20. Bai JZ, Ding WM, Liu ZJ, et al. Transferrin receptor expression of human mesenchymal stem cells and in vitro tracking by autoradiography after transplantation in spinal cord. Beijing Da Xue Xue Bao. 2004;36:276-280.

21. Ray P, De A, Min JJ, Tsien RY, Gambhir SS. Imaging tri-fusion multimodality reporter gene expression in living subjects. Cancer Res. 2004;64:1323-1330.

22. Cao F, Lin S, Xie X, et al. In vivo visualization of embryonic stem cell survival, proliferation, and migration after cardiac delivery. Circulation. 2006;113:1005-1014.

23. Wu JC, Spin JM, Cao F, et al. Transcriptional profiling of reporter genes used for molecular imaging of embryonic stem cell transplantation. Physiol Genomics. 2006;25:29-38.

24. Ponomarev V, Doubrovin M, Serganova I, et al. A novel triple-modality reporter gene for whole-body fluorescent, bioluminescent, and nuclear noninvasive imaging. Eur J Nucl Med Mol Imaging. 2004;31:740-751.

25. Krishnan M, Park JM, Cao F, et al. Effects of epigenetic modulation on reporter gene expression: implications for stem cell imaging. FASEB J. 2006;20:106-108.

26. Zielske SP, Gerson SL. Lentiviral transduction of P140K MGMT into human $\mathrm{CD} 34(+)$ hematopoietic progenitors at low multiplicity of infection confers significant resistance to $\mathrm{BG} / \mathrm{BCNU}$ and allows selection in vitro. Mol Ther. 2002;5:381-387.

27. Lennon DP, Haynesworth SE, Young RG, Dennis JE, Caplan AI. A chemically defined medium supports in vitro proliferation and maintains the osteochondral potential of rat marrow-derived mesenchymal stem cells. Exp Cell Res. 1995; 219:211-222.

28. Bradford MM. A rapid and sensitive method for the quantitation of microgram quantities of protein utilizing the principle of protein-dye binding. Anal Biochem. 1976;72:248-254.

29. Gambhir SS, Bauer E, Black ME, et al. A mutant herpes simplex virus type 1 thymidine kinase reporter gene shows improved sensitivity for imaging reporter gene expression with positron emission tomography. Proc Natl Acad Sci USA. 2000;97:2785-2790.

30. Hruby DE, Ball LA. Cell-free synthesis of enzymatically active vaccinia virus thymidine kinase. Virology. 1981;113:594-601.

31. Tournoy KG, Depraetere S, Pauwels RA, Leroux-Roels GG. Mouse strain and conditioning regimen determine survival and function of human leucocytes in immunodeficient mice. Clin Exp Immunol. 2000;119:231-239.

32. Dennis JE, Haynesworth SE, Young RG, Caplan AI. Osteogenesis in marrowderived mesenchymal cell porous ceramic composites transplanted subcutaneously: effect of fibronectin and laminin on cell retention and rate of osteogenic expression. Cell Transplant. 1992;1:23-32.

33. Alauddin MM, Conti PS. Synthesis and preliminary evaluation of 9-(4- $\left[{ }^{18} \mathrm{~F}\right]-$ fluoro-3-hydroxymethylbutyl)guanine ([ $\left.\left.{ }^{18} \mathrm{~F}\right] \mathrm{FHBG}\right)$ : a new potential imaging agent for viral infection and gene therapy using PET. Nucl Med Biol. 1998; 25:175-180

34. Kim YH, Lee DS, Kang JH, et al. Reversing the silencing of reporter sodium/ iodide symporter transgene for stem cell tracking. J Nucl Med. 2005;46:305311.

35. Su H, Forbes A, Gambhir SS, Braun J. Quantitation of cell number by a positron emission tomography reporter gene strategy. Mol Imaging Biol. 2004;6: 139-148.

36. Dettman GL, Stopeck AT, Meyer GW, Iosiphidis AH. In vivo migration of ${ }^{111} \mathrm{In}$ radiolabeled mouse-spleen lymphocytes and tumor cells. Int J Nucl Med Biol. 1983;10:11-16.

37. Read EJ, Keenan AM, Carter CS, Yolles PS, Davey RJ. In vivo traffic of indium111-oxine labeled human lymphocytes collected by automated apheresis. J Nucl Med. 1990;31:999-1006. 